\title{
Extended glaze firing improves flexural strength of a glass ceramic
}

\author{
Iana L. Aurélio ${ }^{a}$, Sara Fraga ${ }^{a}$, Lucio S. Dorneles ${ }^{b}$, Marco A. Bottino ${ }^{c}$, \\ Liliana G. May ${ }^{d, *}$ \\ a Dental Science Post Graduate Program, Federal University of Santa Maria, Santa Maria, Rio Grande do Sul, Brazil \\ b Department of Physics, Federal University of Santa Maria, Santa Maria, Rio Grande do Sul, Brazil \\ c Department of Dental Materials and Prosthodontics, São Paulo State University, São José dos Campos, São Paulo, \\ Brazil \\ d Department of Restorative Dentistry, Federal University of Santa Maria, Santa Maria, Rio Grande do Sul, Brazil
}

\section{A R T I C L E I N F O}

Article history:

Received 27 January 2015

Received in revised form

18 April 2015

Accepted 24 October 2015

\section{Keywords:}

Glass ceramic

Leucite

CAD/CAM

Machining

Roughness

Thermal cycle

Glaze

Biaxial flexure strength

\begin{abstract}
A B S T R A C T
Objective. To investigate the effect of firing protocols on flexural strength, surface roughness, and crystalline structure of a leucite-based glass ceramic.

Methods. Discs produced by automated machining were distributed into five groups $(n=30)$ according to the applied firing protocols, conducted above $\left(790^{\circ} \mathrm{C}\right)$ or below $\left(575^{\circ} \mathrm{C}\right)$ the ceramic transition temperature $\left(T_{g}\right)\left(625 \pm 20^{\circ} \mathrm{C}\right)$ : C - control, no heat treatment; G790 - glaze firing $\left(790^{\circ} \mathrm{C}\right.$ ) for $1.5 \mathrm{~min}$ (manufacturer-recommended); G790-SC - G790 modified by slow cooling; EGF790-SC - extended G790 for 15 min, with slow cooling; and EF575-SC - extended firing below $\mathrm{T}_{g}$ at $575^{\circ} \mathrm{C}$ for $15 \mathrm{~min}$, with slow cooling. Discs were subjected to biaxial flexural tests and results were assessed using Weibull analysis. Surface roughness was measured before and after treatments. One specimen from each group was used for X-ray diffraction (XRD).

Results. Highest values of characteristic strength $\left(\sigma_{0}\right)$ were obtained for EGF790-SC (211.7 MPa). Regimens EF575-SC, G790-SC, and G790 produced $\sigma_{0}$ values (167.9, 157.7, and 153.7 MPa, respectively) lower than the control (C) $(187.7 \mathrm{MPa})$. The Weibull modulus $(\mathrm{m})$ was statistically similar between groups. All treatments reduced the mean roughness (Ra) of the specimens. Extended cycles (EGF790-SC, EF575-SC) decreased the mean amplitude (Rz). XRD revealed no crystalline phase transformation and slight changes in size of leucite crystallites.

Significance. Increased values of fracture strength and decreased surface roughness for a leucite-reinforced glass ceramic are achieved by extended glaze firing after machining. Crystalline structure is not modified. Glaze cycles, manufacturer-recommended or modified by slow cooling, and firing below $\mathrm{T}_{g}$, significantly reduce fracture strength.
\end{abstract}

() 2015 Academy of Dental Materials. Published by Elsevier Ltd. All rights reserved.

\footnotetext{
* Corresponding author at: Federal University of Santa Maria, Faculty of Odontology, MDS-PhD Graduate Program in Oral Science, Prosthodontics-Biomaterials Units, R. Floriano Peixoto, 1184, 97015-372 Santa Maria, Brazil. Tel.: +55 5532209276 ; fax: +55 55 32209272.

E-mail addresses: lamadridiana@hotmail.com (I.L. Aurélio), sara.odonto@yahoo.com.br (S. Fraga), lucio.dorneles@ufsm.br (L.S. Dorneles), mmbottino@uol.com.br (M.A. Bottino), liligmay@gmail.com (L.G. May).
} 


\section{Introduction}

Clinical studies involving leucite-reinforced glass ceramic restorations report survival rates between 76 and 97\%, with bulk fracture being one of the main reasons for failure of these restorations, totaling $3-11 \%$ of failures [1-4]. Cohesive chipping of the ceramic is mentioned in the literature, but this has not been considered as a failure, since this type of defect does not prevent functioning of the restorations $[1,2,4]$.

Glass ceramics are widely used in indirect restorations that require aesthetic appeal, because they mimic the dental tissues and provide a mechanical strength that is greater than that of feldspathic ceramics. The improvements in the mechanical properties of these materials are a result of the higher content of crystals, such as leucite [5-7], and the development of prefabricated ceramic blocks for CAD-CAM (Computer Aided Design, Computer Aided Machining) systems, produced using a standardized process that results in a more homogeneous material [8,9]. However, even with these fracture toughness advances, the ceramic components maintain their brittle behavior, accumulating residual stresses as a result of the machining process, and are thus susceptible to different failures [10-12]. On the other hand, it is believed that machining might introduce thin compressive stresses to the surface of the ceramic with a positive effect on its strength $[13,14]$. Thus, an understanding of these effects is essential for predicting possible failure mechanisms and assessing the reliability of restorations made by CAD-CAM systems.

Different heat treatments have been employed for glass ceramics in an attempt to control residual stresses $[15,16]$, while potentially reducing some of the damage accumulated during the various steps of fabricating the restorations. These treatments are conducted at temperatures near the glass transition temperature $\left(T_{g}\right)$, where the material viscosity is reduced, and structural rearrangements promote relaxation of internal stresses in the ceramic $[17,18]$. However, cycles that reach temperatures close to the material softening point $[19,20]$, in order to promote healing of possible defects, have also been reported. A relevant aspect in firing treatments for glass ceramics is the way in which the cooling stage is conducted, since it is the rate (fast or slow) at which this process occurs that will determine how much tension will be released or accumulated $[16,21]$. Below $\mathrm{T}_{g}$, the glass behaves as a solid and all structural contraction reverts to residual stress locked inside the material [22]. Higher cooling rates usually result in thermal contraction and solidification being non-uniform because of temperature gradients, leading to the development of stresses [23], and possible weakening of the material.

Materials consisting of different phases, such as leucitereinforced glass ceramics (with two phases, glassy and crystalline), should be treated with extreme caution because these phases behave differently during heat treatment [24-26]. This situation requires thermal schemes that meet the requirements for structural balance between phases. Some studies show that, depending on the regimen adopted, alterations in the crystals, and even in the constituent phases of the materials, may occur $[27,28]$.

There are studies showing that routine laboratory thermal cycles, such as glazing, may be associated with mechanical and structural changes, which could reduce the fracture strength of ceramic materials [11,29-32]. Thus, these treatment schemes must be taken into account when trying to accurately predict the long-term performance of restorations. However, there is inconsistency in the literature regarding different thermal protocols applied to ceramic materials. Existing data on the influence of these treatments on structural and mechanical characteristics of the material are inconclusive.

The present study aimed to investigate the effect of different firing protocols on the fracture strength of a leucitereinforced glass ceramic. The first hypothesis tested was that cycles that adopt heating baths at temperatures above the glass transition temperature, followed by slow cooling, would produce higher values of fracture strength. In addition, we sought to evaluate the effect of these treatments on surface roughness and crystalline structure of the ceramic (crystallite size and phase changes), testing a second hypothesis that presumed increases in strength values would be associated with a reduction in surface roughness. A third hypothesis was that a possible decrease in strength would be associated with an increase in crystallite size and crystal phase changes.

\section{Materials and methods}

\subsection{Machining of ceramic discs by CAD/CAM}

Discs with $13.5 \mathrm{~mm}$ diameter and $1.4 \mathrm{~mm}$ thickness were obtained by automated machining of leucite glass ceramic fully-sintered blocks $(14 \mathrm{~mm} \times 14 \mathrm{~mm} \times 18 \mathrm{~mm}$ ) (IPS Empress CAD C14L, Ivoclar Vivadent AG, Liechtenstein), using a CEREC inLab MC XL milling unit (Sirona Dental Systems Gmbh, Germany). A specific pair of diamond burs for glass ceramics (cylinder pointed bur 12S and step bur 12S, Sirona Dental Systems $\mathrm{Gmbh}$, Germany) was used for machining under intense irrigation. The replacement of the pairs of burs was performed on machine request or failure of a bur.

At the end of the machining process, slight polishing was manually performed, using 400 and 600 grit silicon carbide paper on the upper surface of the discs, to achieve a thickness of $1.4 \mathrm{~mm}( \pm 0.05 \mathrm{~mm})$ and flatten the surface. The bottom side of the discs was kept unchanged (as-machined) and had its surface roughness measured. After that, discs were randomly distributed among the five experimental groups $(n=30$ in each group), using the Random Allocation version 1.0 program (developed by M. Saghaei, Department of Anesthesia, Isfahan University of Medical Sciences, Isfahan, Iran). Statistical analysis was performed in order to validate the randomization. No statically significant difference was found in the initial roughness among the groups ( $p>0.05$ in Kruskal-Wallis test). Four additional discs were used for complementary microstructural analysis.

\subsection{Determination of glass transition temperature $\left(\mathrm{T}_{g}\right)$}

Differential Thermal Analysis (DTA) [33] was performed to estimate the $T_{g}$ of the ceramic. The analysis was performed in a thermogravimetric analysis instrument (Mettler TGA/SDTA $851 \mathrm{e}$ ) with approximately $20 \mathrm{mg}$ of material at a heating rate of $1^{\circ} \mathrm{C} / \mathrm{min}$ from room temperature to $1000^{\circ} \mathrm{C}$, in an oxygen 
Table 1 - Experimental groups and firing protocols.

\begin{tabular}{|c|c|c|c|c|c|}
\hline \multicolumn{6}{|c|}{ Groups $(n=30)$} \\
\hline & $\begin{array}{l}\text { Glaze firing } \\
\left(_{(G 790)^{a}}\right.\end{array}$ & $\begin{array}{c}\text { Glaze firing } \\
\text { modified by slow } \\
\text { cooling (G790-SC) }\end{array}$ & $\begin{array}{c}\text { Extended glaze } \\
\text { firing (EGF790-SC) }\end{array}$ & $\begin{array}{c}\text { Extended firing } \\
\text { below } \mathrm{T}_{g} \\
\text { (EF575-SC) }\end{array}$ & Control (C) \\
\hline Machined & Yes & Yes & Yes & Yes & Yes \\
\hline Initial temperature & $403^{\circ} \mathrm{C}$ & $403^{\circ} \mathrm{C}$ & $403^{\circ} \mathrm{C}$ & $403^{\circ} \mathrm{C}$ & - \\
\hline Temperature increase rate & $100^{\circ} \mathrm{C} / \mathrm{min}$ & $100^{\circ} \mathrm{C} / \mathrm{min}$ & $100^{\circ} \mathrm{C} / \mathrm{min}$ & $100^{\circ} \mathrm{C} / \mathrm{min}$ & - \\
\hline Final temperature & $790^{\circ} \mathrm{C}$ & $790^{\circ} \mathrm{C}$ & $790^{\circ} \mathrm{C}$ & $575^{\circ} \mathrm{C}$ & - \\
\hline Heating bath dwell time & $1.5 \mathrm{~min}$ & $1.5 \mathrm{~min}$ & $15 \mathrm{~min}$ & $15 \mathrm{~min}$ & - \\
\hline Cooling rate & $\begin{array}{l}\text { Fast. Immediate } \\
\text { furnace opening at } \\
\text { the end of heating }\end{array}$ & $\begin{array}{l}\text { Slow. The oven } \\
\text { remained closed } \\
\text { until the material } \\
\text { reached } 200^{\circ} \mathrm{C}\end{array}$ & $\begin{array}{l}\text { Slow. The oven } \\
\text { remained closed } \\
\text { until the material } \\
\text { reached } 200^{\circ} \mathrm{C}\end{array}$ & $\begin{array}{l}\text { Slow. The oven } \\
\text { remained closed } \\
\text { until the material } \\
\text { reached } 200^{\circ} \mathrm{C}\end{array}$ & - \\
\hline Vacuum & Yes & Yes & Yes & Yes & - \\
\hline & From $450^{\circ} \mathrm{C}$ to & From $450^{\circ} \mathrm{C}$ to & From $450^{\circ} \mathrm{C}$ to & From $450^{\circ} \mathrm{C}$ to & \\
\hline & $789^{\circ} \mathrm{C}$, for $3 \min 20 \mathrm{~s}$ & $789^{\circ} \mathrm{C}$, for $3 \mathrm{~min} 20 \mathrm{~s}$ & $789^{\circ} \mathrm{C}$, for $3 \min 20 \mathrm{~s}$ & $575^{\circ} \mathrm{C}$, for $3 \min 20 \mathrm{~s}$ & \\
\hline
\end{tabular}

atmosphere. The $T_{g}$ was determined by the intersection of a line halfway between the two baselines (representing the lower and upper limits of the endothermic reaction) with the tangent at the inflection point of the curve generated by the heat flow [34].

The estimated value of $\mathrm{T}_{g}$ obtained by DTA was consistent with that proposed by the ceramic manufacturer $\left(625 \pm 20^{\circ} \mathrm{C}\right)$ [35]. On this basis, it was possible to establish different firing protocols in relation to $\mathrm{T}_{g}$ and design the experimental groups.

\subsection{Experimental groups: treatments applied to the ceramic}

Experimental groups and firing protocols are described in Table 1. In the control group (C), the specimens were machined but did not receive any heat treatment. In the other groups, the discs were subjected to a thermal schedule performed in a VITA VACUMAT 6000 MP furnace (VITA - Germany).

The thermal cycle for glazing (which we designate as G790), with a mean time of $11 \mathrm{~min}$, strictly follows the manufacturer's recommendations: initial temperature of $403^{\circ} \mathrm{C}$, pre-heating time of $6 \mathrm{~min}$, temperature rise rate of $100^{\circ} \mathrm{C} / \mathrm{min}$, maintenance of temperature at $790^{\circ} \mathrm{C}$ for $1.5 \mathrm{~min}$, and a vacuum environment for $3 \mathrm{~min} 20 \mathrm{~s}$ while the temperature is between $450^{\circ} \mathrm{C}$ and $789^{\circ} \mathrm{C}$ [35]. The furnace opening is located at the end of the heating stage, providing abrupt cooling. The extended schedules (EGF790-SC and EF575-SC) were conducted with the same initial temperature $\left(403^{\circ} \mathrm{C}\right)$, pre-heating time (6 $\mathrm{min})$, and temperature increase rate $\left(100^{\circ} \mathrm{C} / \mathrm{min}\right)$ as the G790 regimen, but were differentiated by holding the specimens in the heating bath for $15 \mathrm{~min}$, either above (EGF790-SC: $790^{\circ} \mathrm{C}$ ) or below (EF575-SC: $575^{\circ} \mathrm{C}$ ) the $\mathrm{T}_{g}$ of the ceramic $\left(625 \pm 20^{\circ} \mathrm{C}\right)$. In both the EGF790-SC and EF575-SC regimens, slow cooling was performed. This stage was characterized by leaving the furnace closed until the material reached $200^{\circ} \mathrm{C}$. The prolonged heating bath, along with the slow cooling, resulted in thermal cycles with a mean duration of $1 \mathrm{~h} 30 \mathrm{~min}$ for group EF575-SC, and $2 \mathrm{~h} 20 \mathrm{~min}$ for group EGF790-SC. The glaze cycle modified by slow cooling (G790-SC) differed from the G790 group only in the slow cooling (to $200^{\circ} \mathrm{C}$ ) step, just described. This modification added just over $1 \mathrm{~h}$ to the total time of the original cycle (group G790).

A vacuum environment was used in order to provide similar conditions for all groups, as glaze firing requires this during a certain part of the cycle. Thus, in the extended regimens, vacuum was applied during the temperature-rise period for the same period of time ( $3 \mathrm{~min} 20 \mathrm{~s}$ ) and from the same temperature $\left(450^{\circ} \mathrm{C}\right)$ as the $\mathrm{G} 790$ and G790-SC groups.

The number of specimens per group subjected to mechanical testing was 30 , so that the material failure probability could be determined and the Weibull modulus calculated for the different treatments. An additional specimen from each group was used for microstructural analysis using X-ray diffraction.

\subsection{Piston-on-three-ball biaxial flexure test}

The fracture strength of the discs was determined using a piston-on-three-ball test, according to ISO 6872/2008 [36], using a universal testing machining (DL-1000 Emic, Brazil). The bottom surface of the disc - the experimental surface - was positioned on three steel spheres $\left(2.5 \mathrm{~mm}\right.$ diameter, $120^{\circ}$ apart and forming a $10 \mathrm{~mm}$ diameter circle), with a load applied at a rate of $0.5 \mathrm{~mm} / \mathrm{min}$, perpendicular to the center of the top surface of the disc, using a cylindrical steel piston with a $1.4 \mathrm{~mm}$ diameter flat tip. A polyethylene strip was placed between the metallic supports and the disc, and between the disc and the piston, in order to uniformly distribute the pressure [37]. The fracture strength, in MPa, was calculated using Eqs. (1)-(3):

$$
\begin{aligned}
& \sigma=\frac{-0.2387 P(X-Y)}{d^{2}} \\
& X=(1+v) \ln \left(\frac{B}{C}\right)^{2}+\left[\frac{1-v}{2}\right]\left(\frac{B}{C}\right)^{2} \\
& Y=(1+v)\left[1+\ln \left(\frac{A}{C}\right)^{2}\right]+(1-v)\left(\frac{A}{C}\right)^{2}
\end{aligned}
$$


where $P$ is the load at fracture $(N), d$ is the disc thickness $(\mathrm{mm})$, $v$ is Poisson's ratio (0.25), A is the support ball radius $(5 \mathrm{~mm})$, $B$ is the radius of the tip of the piston $(0.7 \mathrm{~mm})$ and $C$ is the specimen radius $(6.75 \mathrm{~mm})$.

\subsection{Complementary analyses}

\subsubsection{Measurement of the surface roughness}

The roughness of the bottom surface of the discs was measured after machining (initial roughness) and at the end of each treatment (final roughness), using a contact stylus profilometer (SJ-410, Mitutoyo, Japan).

The Ra (average surface roughness; $\mu \mathrm{m}$ ) and Rz (arithmetic mean peak-to-valley height; $\mu \mathrm{m}$ ) values [38] were determined using the average of three measurements on a path transverse to the machining direction. The roughness measurement protocol is defined in ISO 4287:1997 [39]. A distance scale is established to distinguish waviness from roughness; the sampling length is equivalent to five times a cut-off value $\left(\lambda_{c}\right)$, using the Ra values recorded in initial readings as a reference. The roughness measurements before and after treatment for the other groups were conducted using $\lambda_{\mathrm{c}}=0.8 \mathrm{~mm}$ (the tabulated value for $0.1<\mathrm{Ra} \leq 2.0$ ), resulting in a sampling length of $4 \mathrm{~mm}$. In addition to the use of a cut-off length, a Gaussian filter differentiates between shape defects and the roughness profile.

\subsubsection{X-ray diffraction}

$\mathrm{X}$-ray diffraction (XRD) was performed to characterize the ceramic crystalline structure before and after heat treatment, and to analyze the effect of the firing protocols on the leucite crystallites (sub-micrometer particles).

A disc from each thermal regimen was analyzed by XRD before and after heat treatment (D8 Advance XRD, Bruker AXS $\mathrm{GmbH}$, Germany), using a wavelength of $1.5416 \AA\left(\mathrm{CuK}_{\alpha}\right)$, scan range from $20^{\circ}$ to $45^{\circ}$, step size of $0.009^{\circ}$ and a time per step of $87.5 \mathrm{~s}$. Peaks were identified from the tetragonal leucite values available for powder diffraction patterns (International Centre for Diffraction Data/Joint Committee for Powder Diffraction Studies).

The average size of the crystallites before and after heat treatment was determined from peak (404), located at $2 \Theta \approx 38^{\circ}$ and with FWHM (full width at half maximum) $\approx 0.3^{\circ}$, by the Debye equation, Eq. (4) [40]:

$\mathrm{T}=0.9 \frac{\lambda}{\beta \cos \theta}$

where $\mathrm{T}$ is the crystallite average diameter, $\lambda$ is the $\mathrm{X}$-ray wavelength $(1.5416 \AA), \theta$ is the peak location angle, and $\beta$ is the FWHM of the selected peak, in radians. The real value of $\beta$, free of measurement errors, is calculated using Eq. (5):

$\beta^{2}=\beta_{\mathrm{M}}^{2}-\beta_{\mathrm{S}}^{2}$

where $\beta_{\mathrm{M}}$ is the measured value of $\beta$ for a ceramic specimen and $\beta_{\mathrm{S}}$ is the value obtained with a silicon crystal (crystal size greater than $1000 \AA$ ).

\subsection{Statistical analysis}

The paired t-test (dependent groups) was used to compare the mean surface roughness values (Ra, Rz) intra-groups, before and after each heat treatment, since the data presented a normal distribution ( $p>0.05$ based on the Shapiro-Wilk test).

Weibull statistics $[41,42]$ were used for the analysis of flexural strength, as described by the characteristic strength $\left(\sigma_{0}\right)$, which is the strength at which a cumulative probability of failure of $63.2 \%$ exists for a particular group of test specimens, and the Weibull modulus $(m)$, which represents the variability of the fracture strength value. A small value of $m$ represents a large range of failure strengths in the group, and vice versa. The basic form of the Weibull distribution is shown in Eq. (6):

$P_{f}=1-\exp \left[-\left(\frac{\sigma}{\sigma_{0}}\right)^{m}\right]$

where $P_{f}$ is the cumulative probability of failure and $\sigma$ is the failure stress. From the experimental data, the failure stresses for biaxial flexure within each group were ranked in ascending order, and the probability of failure for each stress $\left(P_{f(\sigma i)}\right)$ was calculated by Eq. (7), where $i$ is the rank order $(1,2,3, \ldots, 30)$ and $N$ is the number of specimens per group:

$P_{f \sigma i}=\frac{i-0.5}{N}$

A rank regression was performed on the bi-axial flexure strength data to estimate the Weibull parameters $\sigma_{0}$ and $m$ [36].

The upper and lower limits of the $95 \%$ confidence intervals for $\sigma_{0}$ and $m$ were calculated according to ENV 843-5:1996 [43]. Differences were considered significant when the confidence intervals failed to overlap.

\section{Results}

The characteristic strength $\left(\sigma_{0}\right)$ and Weibull modulus $(m)$ of the tested groups are given in Table 2. The glazing regimens (G790 and G790-SC) show similar characteristic strengths, but are significantly lower than the control group (C). Extended firing below $\mathrm{T}_{g}$ (EF575-SC) achieves higher strength than both glazing cycles (G790 and G790-SC); however, these values are lower than for the control group (C). Extended glaze firing (EGF790SC) produces the highest flexural strength values, which are greater than those of the other tested groups (G790, G790SC and EF575-SC) and are even significantly higher than the control group (C). The strength values scatter is similar for all groups, since there is no significant difference in $m$ values (Table 2).The intra-group comparisons, between before and after roughness for the same firing treatment, are shown in Table 3. In each group, the heat treated discs served as their own control, before undergoing heat treatment. All heat treatments significantly reduce the mean roughness (Ra) of the specimens. The mean amplitude, represented by the $\mathrm{Rz}$ parameter, presents a statistically significant reduction only for extended firing schedules (EGF790-SC and EF575-SC).The 
Table 2 - Means and 95\% confidence intervals (CI) of characteristic strength $\left(\sigma_{0}\right)$ and the Weibull modulus $(m)$ for the experimental groups.

\begin{tabular}{lccccc}
\hline \multicolumn{7}{c}{ Groups $(n=30)$} \\
\hline C & G790 & G790-SC & EGF790-SC & EF575-SC \\
\hline$\sigma_{0}-\mathrm{MPa}(\mathrm{CI}=95 \%)$ & $187.7(181.1-194.4)^{\mathrm{b}}$ & $153.7(148.8-158.6)^{\mathrm{d}}$ & $157.7(153.3-162.0)^{\mathrm{d}}$ & $211.7(206.7-226.9)^{\mathrm{a}}$ & $167.9(163.0-172.8)^{\mathrm{c}}$ \\
$m(\mathrm{CI}=95 \%)$ & $12.0(8.4-15.5)^{\mathrm{a}}$ & $13.3(9.3-17.1)^{\mathrm{a}}$ & $15.5(10.9-20.0)^{\mathrm{a}}$ & $18.1(12.7-23.2)^{\mathrm{a}}$ & $14.7(10.3-18.9)^{\mathrm{a}}$ \\
\hline \multicolumn{2}{l}{ Different superscripts (a-d) indicate groups with statistically significant differences (95\% CIs do not overlap). } \\
\hline
\end{tabular}

Table 3 - Mean and standard deviation of surface roughness values (Ra and Rz), before and after treatments, and intra-group comparisons (paired t-test at $\mathbf{5} \%$ significance level).

\begin{tabular}{|c|c|c|c|c|c|}
\hline Groups & Treatment stage & $\mathrm{Ra}(\mu \mathrm{m})$ (standard deviation) & $p$-Value ${ }^{a}$ & $\mathrm{Rz}(\mu \mathrm{m})$ (standard deviation) & $p$-Value \\
\hline \multirow{2}{*}{ G790 } & Before & $1.47(0.24)$ & \multirow{2}{*}{0.045} & $9.59(1.73)$ & \multirow{2}{*}{0.377} \\
\hline & After & $1.41(0.24)$ & & $9.24(1.58)$ & \\
\hline \multirow{2}{*}{ G790-SC } & Before & $1.46(0.30)$ & \multirow{2}{*}{0.001} & $9.59(1.45)$ & \multirow{2}{*}{0.160} \\
\hline & After & $1.38(0.29)$ & & $9.05(1.76)$ & \\
\hline \multirow{2}{*}{ EGF790-SC } & Before & $1.54(0.29)$ & \multirow{2}{*}{0.001} & $10.03(1.72)$ & \multirow{2}{*}{0.000} \\
\hline & After & $1.47(0.25)$ & & $9.39(1.45)$ & \\
\hline \multirow{2}{*}{ EF575-SC } & Before & $1.45(0.27)$ & \multirow{2}{*}{0.000} & $9.46(1.58)$ & \multirow{2}{*}{0.001} \\
\hline & After & $1.38(0.28)$ & & $8.97(1.78)$ & \\
\hline
\end{tabular}

$\mathrm{X}$-ray diffraction analysis does not indicate any changes in the crystalline phase of the material after the heat treatments, since the leucite remains in its stable tetragonal crystalline arrangement (Fig. 1).

The sizes of the leucite crystallites, before and after the thermal cycles, determined from the width of XRD peaks, are given in Table 4 . The mean crystallite size, before treatment, is $28.8 \mathrm{~nm}$. The variation with thermal cycle ranges from a reduction on the order of $0.8 \mathrm{~nm}$ for group G790-SC to an increase of $2.1 \mathrm{~nm}$ for group EGF790-SC.

\section{Discussion}

Thermal schemes ranging from routine laboratory procedures, such as glazing, to more complex firing protocols, such as

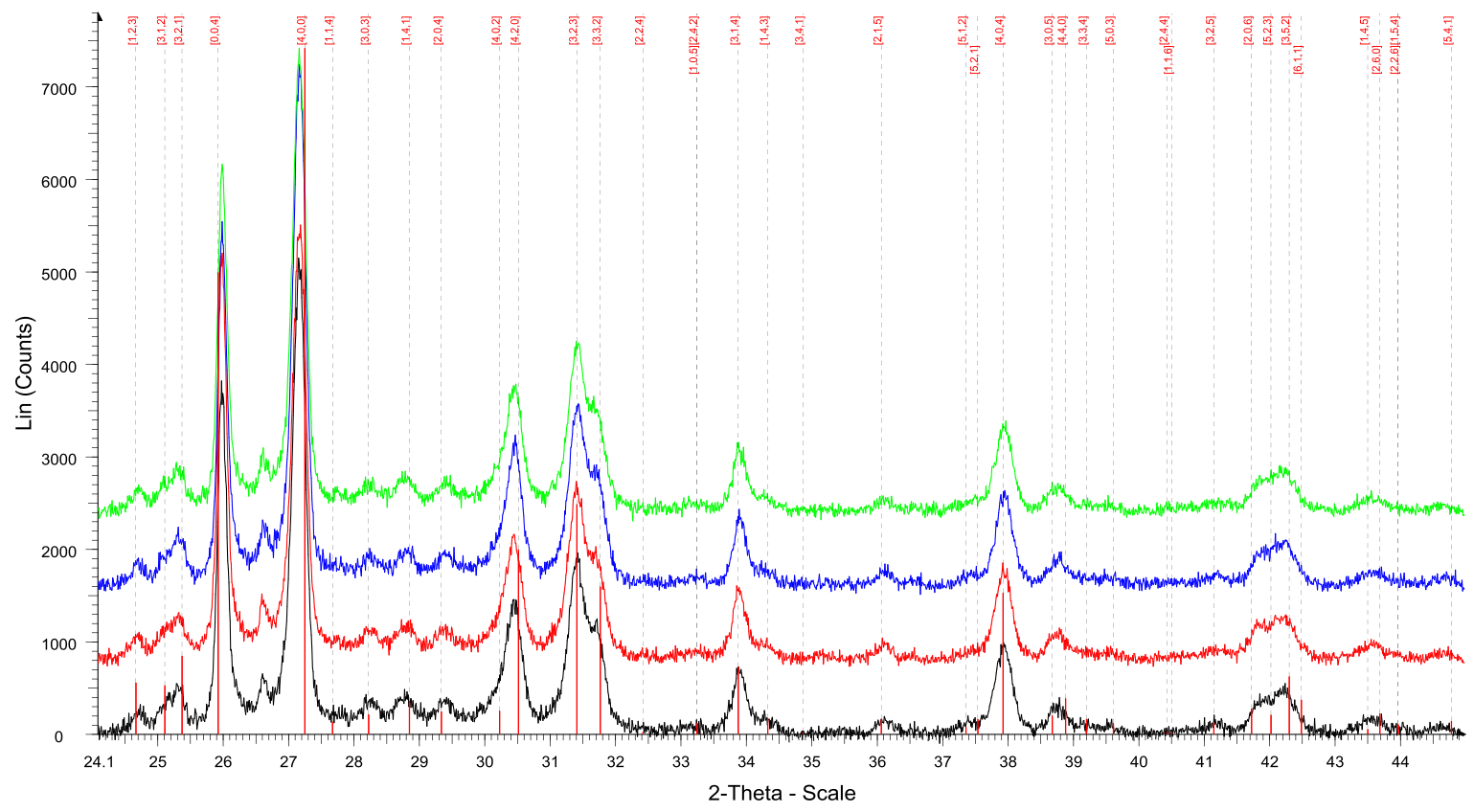

Fig. 1 - X-ray diffraction plot related to cycles: G790 (green), EGF790-SC (blue), EF575-SC (red) and G790-SC (black), after heat treatments. Vertical red lines above the 2-Theta axis indicate the location of peaks for tetragonal leucite in powder diffraction patterns (International Centre for Diffraction Data/Joint Committee for Powder Diffraction Studies). 
Table 4 - Size of the leucite crystallites $(\mathrm{nm})$, before and after heat treatment, and size variations ( $\mathrm{nm}$ and \%) after treatment. Data taken from peak at $2 \theta \sim 37.9^{\circ}$, corresponding to tetragonal leucite (404). (One sample from each treatment group.).

\begin{tabular}{llccrr} 
Peak & Groups & $\begin{array}{c}\text { Crystallite size } \\
\text { before }(\mathrm{nm})\end{array}$ & $\begin{array}{c}\text { Crystallite size } \\
\text { after }(\mathrm{nm})\end{array}$ & $\begin{array}{c}\text { Crystallite size } \\
\text { variation }(\mathrm{nm})\end{array}$ & $\begin{array}{r}\text { Crystallite size } \\
\text { variation }(\%)\end{array}$ \\
\hline \multirow{4}{*}{ (404) } & G790 & 28.7 & 30.7 & 2.0 & 6.6 \\
& G790-SC & 29.1 & 28.3 & -0.8 & -1.7 \\
& EGF790-SC & 28.9 & 31.0 & 2.1 & 7.6 \\
& EF575-SC & 28.6 & 30.1 & 1.5 & 4.5 \\
\hline
\end{tabular}

annealing, have been investigated for possible effects on the mechanical strength of ceramic materials [11,15,29-32] and machined ceramic specimens $[19,20]$. The present study aimed to analyze the effect of firing protocols on flexural strength of a leucite-reinforced glass ceramic. The specimens, with a simplified geometry and produced using automated machining, provided a simulation of the effects of heat treatments on restorations.

The first hypothesis tested was that cycles that adopt heating baths at temperatures above the glass transition temperature followed by slow cooling would produce higher values of fracture strength in the material. The highest value of characteristic strength $\left(\sigma_{0}=211.7 \mathrm{MPa}\right)$ was achieved by the EGF790-SC schedule, in agreement with the first hypothesis. This scheme (EGF790-SC) appears to have promoted a favorable stress state, since its mean value of biaxial flexural strength $(211.7 \mathrm{MPa})$ was larger than that reported by the manufacturer (160 MPa) [44] and was also higher than that obtained for the control group (187.7 MPa). With the extended dwell time (15 min) at $790^{\circ} \mathrm{C}$, a layer of lower thermal expansion glass may be formed in the ceramic surface, placing the outer surface in compression when cooled. Existing compressive stress offsets some of the local tensile stress at surface flaws produced by applied loading, and greater loading is needed to initiate flaw propagation from the external surface $[29,30,32]$. This condition overcomes any possible mechanical disadvantages associated with the prior relief of compressive stresses during machining $[13,14]$. Furthermore, in the EGF790-SC cycle, the temperature was decreased carefully to avoid development of stresses produced by the non-uniform thermal contraction and solidification that results from temperature gradients [23]. For ceramic materials, strength increases with decreasing flaw depth and sharpness. During the extended glaze firing, the depth and shape of cracks on the ceramic surface may have changed $[19,29,30,45]$, with the crack tips changing from sharp to round, increasing the stress needed for crack initiation. Therefore, the similar values of the Weibull modulus $(m)$ found among groups (Table 2) indicate that the distribution of defects in the material after the heat treatments was not modified by any of those cycles.

In the G790-SC regimen, the ceramic was held at $790^{\circ} \mathrm{C}$ for $1.5 \mathrm{~min}$, as suggested by the glazing protocols for this ceramic [35], while in EGF790-SC the ceramic was maintained at this temperature for $15 \mathrm{~min}$. In both groups, slow cooling rates were then applied. Therefore, these regimens differ only in the period that the material remained at $790^{\circ} \mathrm{C}$. However, the G790-SC group does not present an improvement in fracture strength, contradicting the first hypothesis of the study. The strength values for the G790-SC group were about 157.7 MPa, which were significantly lower when compared to those of group EGF790-SC (211.7 MPa). These findings suggest that the short dwell time $(1.5 \mathrm{~min})$ at $790^{\circ} \mathrm{C}$ of the G790-SC schedule is insufficient for the achievement of the optimal conditions of viscosity to promote a surface layer with a favorable stress state and modification of defects on the ceramic surface. Denry [10] indicated that sub-surface and surface defects produced during the milling stage might not be minimized after glazing and eventually become the origin of fractures of ceramic restorations after some time in service. Therefore, the selection of a proper temperature range and dwell time [46] for the glazing of machined glass ceramic restorations is required to promote a beneficial stress state, with the formation of a compressive surface layer, and even to induce some crack-shape modification.

The period for which the material remains heated to a constant temperature also appears to be critical when the procedure is carried out below the $\mathrm{T}_{g}$, as with the $575^{\circ} \mathrm{C}$ used in the EF575-SC cycle, which presented lower flexural strength values (Table 2) when compared to the EGF790-SC and C groups. The ceramics subjected to this regimen (EF575-SC) apparently remained at that temperature for an insufficient period during the heating step, not allowing an efficient reduction in glass matrix viscosity to induce modification of surface defects or a favorable final stress state. At $575^{\circ} \mathrm{C}$ (below $\mathrm{T}_{g}$ ) molecular movements are very slow during the glass matrix solidification process [16], hindering stress adjustments and preventing surface flaw modification. According to Bertolotti and Shelby [47], for the glass to release tension, an extended heating time is required to compensate for the use of a temperature below the glass transition; a similar result was seen by Fischer et al. [15], where a firing time of $10 \mathrm{~h}$ below $\mathrm{T}_{g}$ was necessary to achieve a relaxation of tensions and an increase in strength of the ceramic.

Another plausible explanation for the reduction in the strength values (Table 2) in the EF575-SC, G790 and G790-SC groups is that the firing protocols used, with a lower temperature and/or shorter heating baths, might have promoted only the relief of surface compressive stresses caused by machining [12]. According to Marshall et al. [13], machining introduces a thin strip of compressive stress on the ceramic surface, which provides a positive effect on strength. Giordano, Cima and Pober [11] observed a reduction in the flexural strength of a conventional feldspathic ceramic after heat treatment, which was attributed to the reduction of compressive stresses introduced by polishing. Also, the depth and shape of surface cracks may have remained unaltered, leading to lower strength.

As outlined in the above discussion, both heating bath temperature and dwell time influence the flexural strength of the glass ceramic. However, slow cooling seems not to 
improve flexural strength without sufficient periods of heating, regardless of being above or below the $\mathrm{T}_{g}$ of the glass ceramic $\left(625 \pm 20^{\circ} \mathrm{C}\right)$. Examples are the G790-SC group $\left(790^{\circ} \mathrm{C}\right)$, having the bath above $\mathrm{T}_{g}$ but without extended dwell time, and the EF575-SC group $\left(575^{\circ} \mathrm{C}\right)$, having extended dwell time but below $T_{g}$. This indicates that the slow cooling process, although important in some studies $[48,49]$, has a positive effect on the fracture strength of the glass ceramic after the machining process only when associated with adequate temperature and dwell time of the heating bath.

In parallel to the strength studies, we investigated the effect of heat treatments on the ceramic surface roughness. Table 3 compares the surface roughness of each group of discs between its machined-only and heat treated states. The second hypothesis tested is whether the increase in strength would be associated to a reduction in surface roughness within a same group, as a result of heat treatment. The EGF790-SC scheme shows improved strength values (211.7 MPa); it also produces significantly lower values of surface roughness $\left(\mathrm{Ra}_{\mathrm{after}}=1.47 \mu \mathrm{m} ; \mathrm{Rz}_{\mathrm{after}}=9.39 \mu \mathrm{m}\right)$ in comparison to the same discs prior to treatment $\left(\operatorname{Ra}_{\text {before }}=1.54 \mu \mathrm{m}\right.$; $R z_{\text {before }}=10.03 \mu \mathrm{m}$ ), supporting the second hypothesis. However, this behavior is not as clearly demonstrated in the other groups. The G790 and G790-SC treatments reduce the mean surface roughness (Ra) of the ceramic with statistical significance, but changes in the mean amplitude of the profile $(\mathrm{Rz})$ do not reach significance, and these treatments produce the lowest material strengths. The EF575-SC cycle significantly reduces both the Ra and $\mathrm{Rz}$ parameters, but this improvement in the roughness profile is not accompanied by improved strength values. Thus, the second hypothesis is partially rejected because the fracture strength of the ceramic decreases, despite a reduction in surface roughness for the EF575-SC, G790 and G790-SC groups. It appears that, despite statistical significant, the reduction of surface roughness of these levels $(\sim 5 \%)$ for all groups was not pratically significant and does not play an important role in strength, as machining microcracks seem to do.

Few studies have investigated the possibility that ceramics submitted to heat treatment might present changes in their roughness parameters and healing of defects. Lenz et al. [45] suggested that, at temperatures that approximate $T_{g}$, the viscoelastic behavior of the porcelain may favor the closure of microcracks and produce higher mechanical strength values. In the study proposed by Addison et al. [20], the surface roughness of disks made from a feldspathic ceramic machined by a CAD/CAM system (Vita Mark II) is not influenced by annealing $\left(900^{\circ} \mathrm{C}\right.$ for $\left.1 \mathrm{~h}\right)$, even though this value is very close to the softening temperature of the material $\left(914 \pm 8^{\circ} \mathrm{C}\right)$. However, when this ceramic is subjected to the same temperature $\left(900^{\circ} \mathrm{C}\right)$ for a period of $2 \mathrm{~h}$, a reduction in the size of the defect generated by Vickers indentation in the specimens is detected, indicating possible surface modification, but is not followed by an increase in strength of the material [19]. SEM analysis of specimens obtained from different machinable ceramics (Vita Mark II, PROCAD, IPS Empress CAD and IPS e.max CAD) reveals that, independent of the tested ceramic, the glazed surfaces are homogeneously smooth in comparison to the ribbed surfaces of polished samples. However, glazing produces strength values that are statistically similar to those produced by the polished groups [50]. A complementary analysis using electron microscopy to investigate possible healing of defects by heat treatments may help clarify this issue. This type of analysis requires very well-polished specimens in order to observe details of cracks before and after heat treatment. However, this is not feasible in machined specimens due to the large topographical changes caused by machining. Furthermore, the effect of machining explored in this study could be influenced by the necessary polishing procedures.

$\mathrm{X}$-ray diffraction was used to investigate the microstructural changes caused by the thermal regimen for each treated glass ceramics group. Material properties, such as flexural strength, wear, and aesthetic characteristics, can be highly dependent on crystal structure [18] and crystallite size [51]. Thus, control of the crystalline structure, crystalline phases, and crystallite size (degree of crystallization) are concerns when handling a ceramic material, for instance when the ceramic is subjected to a firing protocol.

Typically, crystallite size tends to increase with an increase of treatment temperature, presenting a narrowed X-ray diffraction peak. When a peak is broadened, a reduction of particle size is normally inferred [52]. However, a temperature increase generated by the tested cycles did not cause changes in the degree of crystallization of the material, reflecting little significant change in crystallite size (Table 4) at the evaluated peak (404), which did not suffer from narrowing or enlargement (Fig. 1). The diffraction peak chosen for this analysis was (404), as a peak located at a smaller $2 \Theta$ would introduce a greater error in the extracted parameters [40]. Some studies show that, depending on the regimen adopted, alterations in the constituent phases of the materials may occur $[27,28]$. In the present work, none of the heat treatments results in changes to the crystalline phase of the material, with the leucite remaining in its stable tetragonal form (Fig. 1). Thus, the third hypothesis, that possible decreases in strength values are related to changes in the original crystalline structure, such as phase changes and increase in crystallite size, is rejected.

The fact that no thermal regimens cause changes in the crystalline microstructure of the material shows that the cycles do not change the composition set by the manufacturer. The relevance of this information is mainly associated with the EGF790-SC cycle, which improves the fracture strength of the glass ceramic without changing its original structure. It is noteworthy that small variations in composition and microstructure may provide new mechanical, chemical and physical properties of a material [18]. The improvement in fracture strength achieved by this firing (EGF790-SC) appears to be related to achieving a favorable final stress state, reducing the surface roughness values and possibly modifying the shape of cracks on the ceramic surface by heat treatment.

The finishing stages of glass ceramic restorations, which frequently include the application of stain oxides and glazing, are important for mimicking tooth structure. Based on the current results, the possible deleterious effect of the glazing cycle on the fracture strength of leucite-based ceramics suggests the need for studies evaluating the feasibility of employing alternative thermal regimens, such as the EGF790-SC cycle - which uses the same temperature suggested by the manufacturer $\left(790^{\circ} \mathrm{C}\right)$, but an extended period of heating $(15 \mathrm{~min})$, followed 
by slow cooling - for glazing restorations. Studies evaluating the effect on the ceramic's optical properties of the EGF790-SC treatment, as a feasible alternative glazing cycle, are needed. Also, studies addressing the fatigue life of the ceramic subjected to these extended firing regimes should be conducted.

\section{Conclusions}

The investigated extended glaze firing treatment improves fracture strength, decreases the surface roughness, and does not modify the original crystalline structure of a hard-machined leucite-reinforced glass. Glaze cycles, manufacturer-recommended or modified by slow cooling, and firing below $\mathrm{T}_{g}$, significantly reduce fracture strength.

\section{R E F E R E N C E S}

[1] Van Dijken JW, Hasselrot L. A prospective 15-year evaluation of extensive dentin-enamel-bonded pressed ceramic coverages. Dent Mater 2010;26:929-39.

[2] Frankenberger R, Taschner M, Garcia-Godoy F, Petschelt A, Krämer N. Leucite-reinforced glass ceramic inlays and onlays after 12 years. J Adhes Dent 2008;10:393-8.

[3] Fradeani M, Redemagni M. An 11-year clinical evaluation of leucite-reinforced glass-ceramic crowns: a retrospective study. Quintessence Int 2002;33:503-10.

[4] Guess PC, Selz CF, Steinhart YN, Stampf S, Strub JR. Prospective clinical split-mouth study of pressed and CAD/CAM all-ceramic partial-coverage restorations: 7-year results. Int J Prosthodont 2013;26:21-5.

[5] Gonzaga CC, Cesar PF, Miranda Jr WG, Yoshimura HN. Slow crack growth and reliability of dental ceramics. Dent Mater 2011;27:394-406.

[6] Cesar PF, Yoshimura HN, Miranda Júnior WG, Okada CY. Correlation between fracture toughness and leucite content in dental porcelains. J Dent 2005;33:721-9.

[7] Song XF, Yin L. Stress and damage at the bur-prosthesis interface in dental adjustments of a leucite-reinforced glass ceramic. J Oral Rehabil 2010;37:680-91.

[8] Giordano R. Materials for chairside CAD/CAM-produced restorations. J Am Dent Assoc 2006;137(Suppl.):14S-21S.

[9] Li RW, Chow TW, Matinlinna JP. Ceramic dental biomaterials and CAD/CAM technology: state of the art. J Prosthodont Res 2014;58:208-16.

[10] Denry I. How and does fabrication damage adversely affect the clinical performance of ceramic restorations? Dent Mater 2013;29:85-96.

[11] Giordano R, Cima M, Pober R. Effect of surface finish on the flexural strength of feldspathic and aluminous dental ceramics. Int J Prosthodont 1995;8:311-9.

[12] Zhang GM, Satish KG, Ko WK. The mechanics of material removal mechanisms in machining ceramics. Technical Report TR 94-22rl. Maryland: Institute for Systems Research (NIST); 1994.

[13] Marshall DB, Evans AG, Khuri Yakub BT, Tien JW, Kino GS. The nature of machining damage in brittle materials. Proc $\mathrm{R}$ Soc Lond A 1983;385:461-75.

[14] Garvie CR, Hannink HR, Pascoe TR. Ceramic Steel? Nature 1975;258:703-4.

[15] Fischer H, Hemelik M, Telle R, Marx R. Influence of annealing temperature on the strength of dental glass ceramic materials. Dent Mater 2005;21:671-7.

[16] Taskonak B, Borges GA, Mecholsky Jr JJ, Anusavice KJ, Moore BK, Yan J. The effects of viscoelastic parameters on residual stress development in a zirconia/glass bilayer dental ceramic. Dent Mater 2008;24:1149-55.

[17] Preston FW. The fundamental law of annealing. Trans Opt Soc $1925 ; 26: 270-3$

[18] Callister WD, Rethwisch DG. Materials science and engineering: an introduction. 8th ed. Wiley; 2009.

[19] Denry IL, Holloway JA, Tarr LA. Effect of heat treatment on microcrack healing behavior of a machinable dental ceramic. J Biomed Mater Res 1999;48:791-6.

[20] Addison O, Cao X, Sunnar P, Fleming GJP. Machining variability impacts on the strength of a "chair-side" CAD-CAM ceramic. Dent Mater 2012;28:880-7.

[21] Taskonak B, Mecholsky Jr JJ, Anusavice KJ. Residual stresses in bilayer dental ceramics. Biomaterials 2005;26:3235-41.

[22] Belli R, Frankenberger R, Appelt A, Schmitt J, Baratieri LN, Greil P, et al. Thermal-induced residual stresses affect the lifetime of zirconia-veneer crowns. Dent Mater 2013;29:181-90.

[23] Benetti P, Kelly JR, Sanchez M, Della Bona A. Influence of thermal gradients on stress state of veneered restorations. Dent Mater 2014;30:554-63.

[24] Serbena FC, Zanotto ED. Internal residual stresses in glass-ceramics: a review. J Non-Cryst Solids 2012;358:975-84

[25] Mackert Jr JR, Rueggeberg FA, Lockwood PE, Evans AL, Thompson WO. Isothermal anneal effect on microcrack density around leucite particles in dental porcelain. J Dent Res 1994;73:1221-7.

[26] Mackert Jr JR, Butts MB, Fairhurst CW. The effect of the leucite transformation on dental porcelain expansion. Dent Mater 1986;2:32-6.

[27] Mackert Jr JR, Russell CM. Leucite crystallization during processing of a heat-pressed dental ceramic. Int J Prosthodont 1996;9:261-5.

[28] Barreiro MM, Vivente EE. Kinetics of isothermal phase transformations in dental porcelain. J Mater Sci Mater Med 1993:4:431-6.

[29] Fairhurst CW, Lockwood PE, Ringle RD, Thompson WO. The effect of glaze on porcelain strength. Dent Mater 1992;8:203-7

[30] Griggs JA, Thompson JY, Anusavice KJ. Effects of flaw size and auto-glaze treatment on porcelain strength. J Dent Res 1996;75:1414-7.

[31] Brackett SE, Leary JM, Turner KA, Jordan RD. An evaluation of porcelain strength and the effect of surface treatment. J Prosthet Dent 1989;61:446-51.

[32] Dong JK, Luthy H, Wohlwend A, Schärer P. Heat-pressed ceramics: technology and strength. Int J Prosthodont 1992;5:9-16.

[33] Klančnik G, Medved J, Mrvar P. Differential thermal analysis (DTA) and differential scanning calorimetry (DSC) as a method of material investigation. RMZ - M\&G 2010;57:127-42.

[34] Rieger J. The glass transition temperature Tg of polymers comparison of the values from differential thermal analysis (DTA DSC) and dynamic mechanical measurements (torsion pendulum). Polym Test 2001;20:199-204.

[35] Ivoclar Vivadent. IPS Empress ${ }^{\circledR}$ CAD: instructions for use labside; 2006.

[36] 6872 ISO. Dentistry - ceramic materials. 3rd ed. Geneva: International Organization for Standardization; 2008.

[37] Wachtman Jr JB, Capps W, Mandel J. Biaxial flexure tests of ceramic substrates. J Mater 1972;7:188-94.

[38] Whitehouse D. Surfaces and their measurement. 1st ed. Hermes Penton Science; 2002

[39] ISO 4287. Geometrical product specifications (GPS) - surface texture: profile method, terms definitions and surface texture parameters. Geneva: International Organization for Standardization; 1997. 
[40] Cullity BD. Elements of X-ray diffraction. 2nd ed. Addison-Wesley Publishing Company; 1978.

[41] Weibull W. A statistical theory of the strength of materials. Swed Inst Eng Res 1939;151:1-45.

[42] Weibull W. A statistical distribution of wide applicability. J Appl Mech 1951;18:293-7.

[43] ENV 843-5. Advanced technical ceramics - monolithic ceramics - mechanical tests at room temperature - Part 5: Statistical analysis. European Standards; 1996.

[44] Ivoclar Vivadent. IPS Empress ${ }^{\circledR}$ CAD: scientific documentation; 2006.

[45] Lenz J, Thies M, Wollwage P, Schweizerhof K. A note on the temperature dependence of the flexural strength of porcelain. Dent Mater 2002;18:558-60.

[46] Kingery WD, Bowen HK, Uhlmann DR. Introduction to ceramics. 2nd ed. Wiley; 1976.

[47] Bertolotti RL, Shelby JE. Viscosity of dental porcelain as a function of temperature. J Dent Res 1979;58:2001-4.
[48] Tholey MJ, Swain MV, Thiel N. Thermal gradients and residual stresses in veneered Y-TZP frameworks. Dent Mater 2011;27:1102-10.

[49] Choi JE, Waddell JN, Swain MV. Pressed ceramics onto zirconia. Part 2: Indentation fracture and influence of cooling rate on residual stresses. Dent Mater 2011;27: 1111-8.

[50] Asai T, Kazama R, Fukushima M, Okiji T. Effect of overglazed and polished surface finishes on the compressive fracture strength of machinable ceramic materials. Dent Mater J 2010;29:661-7.

[51] Theocharopoulos A, Chen X, Wilson RM, Hill R, Cattell MJ. Crystallization of high-strength nano-scale leucite glass-ceramics. Dent Mater 2013;29:1149-57.

[52] Uvarov V, Popov I. Metrological characterization of X-ray diffraction methods for determination of crystallite size in nano-scale materials. Mater Charact 2013;85:111-23. 